\title{
Doppler Information Optimization through Fusion Algorithms
}

\author{
J. Valarmathi \\ Faculty, School of Electronics \\ Engineering \\ VIT University, Vellore
}

\author{
D. S. Emmanuel \\ Faculty, School of Electronics \\ Engineering \\ VIT University, Vellore
}

\author{
S. Christopher \\ CABS, Ministry of Defense, \\ Bangalore, India
}

\begin{abstract}
This paper analyses the velocity estimation of a target, from the Doppler filter using 1) Kalman filter 2) Adaptive Kalman filter 3) Kalman filter with state vector fusion 4) Adaptive Kalman filter with state vector fusion 5) State vector fused adaptive Kalman filter. Simulation through MATLAB gave good response for $4^{\text {th }}$ and $5^{\text {th }}$ algorithms under low signal to noise ratio. $2^{\text {nd }}$ and $3^{\text {rd }}$ algorithms gave better results in intensive maneuvers. But $1^{\text {st }}$ algorithm even though it is low cost and faster, fails due to the delay in response.
\end{abstract}

\section{Keywords}

Adaptive Doppler Kalman filter, state vector fusion, intensive maneuver.

\section{INTRODUCTION}

For tracking the velocity of a target, required Doppler frequency shift of the received echo signal is obtained using short time Fourier transformation (STFT) [4] on a rectangular window function. Then quadratic interpolation is used to find better frequency shift. This estimated velocity is optimized using fusion technique. The integration or fusion of redundant information can reduce overall uncertainty and thus serve to increase the accuracy with which the features are perceived by the system [10]. Multiple sensors providing redundant information can also serve to increase reliability in the case of sensor error or failure [5]. Papic et al., [1] estimated the velocity through window width adaptation, based on the estimates of target acceleration and signal-to-noise ratio. Banerjee et al., [9] and Bhattacharya et al., [3] used the state-vector fusion technique for estimating the position of the target. Hedrick [5] in his research report used adaptive Kalman estimation but used PDA method to obtain fused velocity estimates. In this paper an attempt is made to compare the performance of the state vector fused adaptive Kalman velocity estimation [fig.5] with the adaptive fused Kalman velocity estimation [fig.6].

Here the estimated velocity from Doppler filter is optimized using five different approaches namely 1) Kalman filter [10] 2) Adaptive Kalman filter [1,5] 3) Kalman filter with state vector fusion [2,3,9] 4) Adaptive Kalman filter with state vector fusion $[1,3,5,9] 5)$ State vector fused adaptive Kalman filter and are simulated through MATLAB and compared.
The structure of this paper is as follows. The problem formulation of state vector fusion [2, 3], in Kalman filter structure and the adaptive Kalman filter structure along with the importance of multi sensor data fusion are explained in sections 2 to 6 . In section 7 results of velocity estimation through these algorithms is simulated. Finally the conclusion is given in section 8 .

\section{KALMAN FILTER VELOCITY ESTIMATION}

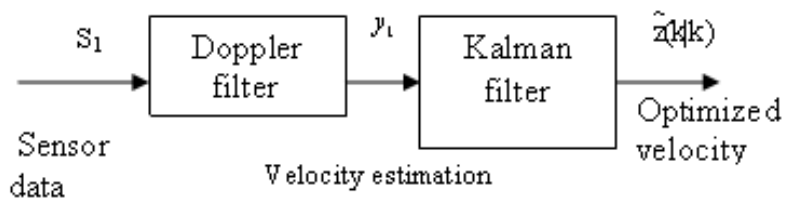

Fig.1 Block diagram of Kalman filter velocity estimation

\subsection{Doppler filter}

Since the target is moving, the reflected signal frequency is different from that of transmitted signal by the Doppler effect $[1,4,11]$. This approximate Doppler frequency shift is obtained from the received signal using Quadratic interpolation of the time dependent Fourier transformation (TDFT) [4] and then smoothed [1,9] using Kalman filter as shown in Fig 1.

\subsection{TDFT}

Also referred to as STFT [4], [1] of a signal x[n], it is defined as $X[n, \lambda)=\sum_{m=-\infty}^{+\infty} x[n+m] w[m] e^{-j \lambda m}$

where $w[m]$ is a window sequence. Taking finite duration of sequences in real time application, STFT [4] becomes,

$X[n, \lambda)=\sum_{m=0}^{N W-1} x[n+m-N W+1] w[m] e^{-j \lambda\left(\frac{2 p}{N}\right) k m}$

where NW represents the window function length, $\mathrm{k}$ is the frequency index that corresponds to the frequency $f_{1}=\frac{k}{T N}$,

$\mathrm{k}=0,1 \ldots \mathrm{N}-1$ and $n$ is the time index that corresponds to the sampling period nT. So, NW sample data window can be used 
together with large $\mathrm{N}$ and the zero-padded FFT may be computed, to minimize errors in the frequency estimation.

\subsection{Quadratic interpolation}

In order to avoid errors due to frequency quantization $[1,9]$ a simple interpolation procedure based on the second order polynomial approximation is used. Assume $f_{p}, f_{q}$ and $f_{r}$ are the highest peaks in the frequency distribution from TDFT analysis. The magnitude of the signal at these frequencies given by

$$
\begin{aligned}
|X[n, p]| & =\max _{0 \leq g \leq((N-1) / 2)}|X[n, g]| \\
|X[n, q]| & =\max _{0 \leq g \leq((N-1) / 2) g \neq p}|X[n, g]| \\
|X[n, r]| & =\max _{0 \leq g \leq((N-1) / 2) g \neq p, g \neq q}|X[n, g]|
\end{aligned}
$$

leads to forming the quadratic equations as in (2)

$a f_{p}^{2}+b f_{p}+c=|X(n, p)|$

$a f_{q}^{2}+b f_{q}+c=|X(n, q)|$

$a f_{r}^{2}+b f_{r}+c=|X(n, r)|$

The Doppler frequency $\hat{f}$, which is the root of the quadratic equation, is estimated as [9],

$\hat{f}=\frac{-b}{2 a}$

Target echo frequency $f_{t}$ is a function of radar transmit frequency $f_{0}$ and radar Doppler frequency may be given by $f_{t}=f_{0} \pm \hat{f}$

where Doppler frequency $\hat{f}$ is a function of radar transmit frequency $f_{0}$ and target velocity $\mathrm{v}_{\mathrm{t}}$. It is positive $(+)$ for approaching targets and negative (-) for receding targets.

$$
\hat{f}=\frac{2 v_{t} f_{0}}{c}
$$

where $c$ is the velocity of light. Substituting equation (5) in equation (4) yields

$$
f_{t}=f_{0}+\frac{2 v_{t} f_{0}}{c}
$$

\subsection{Kalman filter}

Here Kalman filter is used to smooth noisy measurement from the Doppler filter. It involves the design of the Kalman filter whose inputs are the velocity estimates by the Doppler filter and the outputs are the refined velocity estimates $[1,9]$. The state space representation of object kinematics is given by

$\boldsymbol{Z}(\boldsymbol{k})=\emptyset(\boldsymbol{k}) \boldsymbol{Z}(\boldsymbol{k}-\mathbf{1})+\omega(k)$

Where $\boldsymbol{Z}(\boldsymbol{k})$ is the state vector[ $v(k) \quad a(k)]^{\prime}, v(k)$ and $a(k)$ are the velocity and acceleration of the target respectively. $\phi(\mathrm{k})$ is the state transition matrix $\left|\begin{array}{ll}1 & T \\ 0 & 1\end{array}\right|$. Assume the measurement equation is in the following algebraic form $\boldsymbol{Y}(\boldsymbol{k})=\boldsymbol{H} \boldsymbol{Z}(\boldsymbol{k})+\mu(k)$ where $\boldsymbol{H}=\left[\begin{array}{ll}1 & 0\end{array}\right]$

The random variables $\omega(k)$ and $\mu(k)$ represent the process and measurement noise which are white Gaussian with covariance matrices $Q(k)$ and $R(k)$ respectively. The Kalman filter estimates a state at some point of time and then obtains feedback in the form of noisy measurement. These two steps are called prediction and correction.

\subsubsection{Prediction}

$\widehat{Z}(\boldsymbol{k} / \boldsymbol{k}-\mathbf{1})=\emptyset(\boldsymbol{k}) \widehat{\boldsymbol{Z}}(\boldsymbol{k}-\mathbf{1} / \boldsymbol{k}-\mathbf{1})+\omega(k)$

$\boldsymbol{P}(\boldsymbol{k} / \boldsymbol{k}-\mathbf{1})=\emptyset(\boldsymbol{k}) \boldsymbol{P}(\boldsymbol{k}-\mathbf{1} / \boldsymbol{k}-\mathbf{1}) \emptyset(\boldsymbol{k})^{\prime}+Q(k)$

2.4.2 Correction

$K(k)=\boldsymbol{P}(\boldsymbol{k} / \boldsymbol{k}-\mathbf{1}) \boldsymbol{H}^{T}\left[\boldsymbol{H} \boldsymbol{P}(\boldsymbol{k} / \boldsymbol{k}-\mathbf{1}) \boldsymbol{H}^{T} \boldsymbol{R}(\boldsymbol{k})\right]^{-1}$

$\widehat{\boldsymbol{Z}}(\boldsymbol{k} / \boldsymbol{k})=\widehat{\boldsymbol{Z}}(\boldsymbol{k} / \boldsymbol{k}-\mathbf{1})+K(k)[\boldsymbol{Y}(\boldsymbol{k})-\boldsymbol{H} \widehat{\boldsymbol{Z}}(\boldsymbol{k} / \boldsymbol{k}-\mathbf{1})]$

$\boldsymbol{P}(\boldsymbol{k} / \boldsymbol{k})=\boldsymbol{P}(\boldsymbol{k} / \boldsymbol{k}-\mathbf{1})-K(k) \boldsymbol{H} \boldsymbol{P}(\boldsymbol{k} / \boldsymbol{k}-\mathbf{1})$

Where $\boldsymbol{P}(\boldsymbol{k} / \boldsymbol{k}-\mathbf{1})$ and $\boldsymbol{P}(\boldsymbol{k} / \boldsymbol{k})$ are the prediction and correction errors. The first task during the correction step is to compute the Kalman filter gain $K(k)$ from (10). The next step is to generate a posteriori state estimate $\widehat{\boldsymbol{Z}}(\boldsymbol{k} / \boldsymbol{k})$ whose elements are the unknown target velocity and acceleration, by incorporating the measurement as in equation (8). The final step is to obtain an a posteriori error covariance estimate via equation (12).

\section{ADAPTIVE KALMAN FILTER STRUCTURE WITH SINGLE SENSOR}

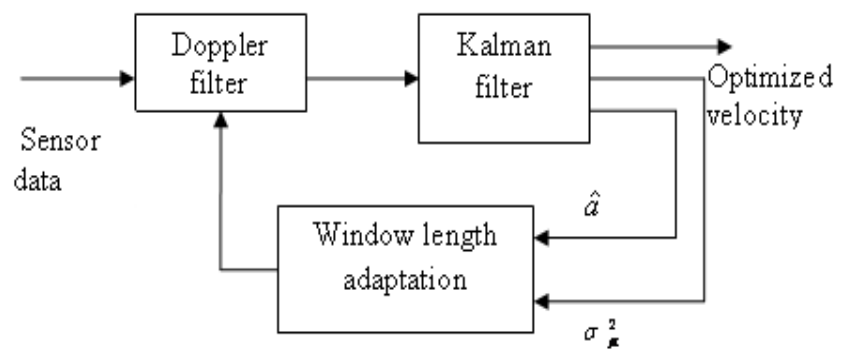

Fig. 2 Block diagram of Adaptive Doppler Kalman filter structure with single sensor

The filter structure as shown in Fig. 2 uses a single sensor. The basic operations in Doppler filter and Kalman filter are the same as in section 2 .

\subsection{Window length adaptation}

Since we are considering fixed window length in Doppler Kalman filter, it experiences a delay in tracking the target dynamics $[1,5,9]$. The applied window function length must be short enough to avoid the effect of velocity averaging across a long interval. This velocity averaging would result in bad velocity estimation in the case of intensive object dynamics resulting in larger acceleration. On the other hand window length has to be long enough to contain relevant information on the Doppler frequency shift especially in the case of low signal to noise ratio. This is in accordance with the known results from the literature $[1,7,8]$. So in adaptive Kalman filtering, proper window length is chosen based on acceleration of the target and SNR. By obtaining $\widehat{\boldsymbol{Z}}(\boldsymbol{k} / \boldsymbol{k})$ the target velocity and acceleration are estimated. Now the next unknown is the SNR which can be estimated as follows. 


\subsection{Choice of optimal window length}

Window length which has less cumulative estimation error (cee) is taken as [1,9] the optimal window length. Thus, Cumulative estimation error (cee) is given as,

$$
\operatorname{cee}(k)=\frac{1}{k} \sum_{j=1}^{k} \frac{|v(j)-\hat{v}(j)|}{|v(j)|}
$$

Cumulative error is estimated (cee) for the set of estimated velocity at a different window length for the estimated SNR. Window length corresponds to the lowest cee is taken as an optimal window length.

\subsection{Signal to noise ratio(SNR) estimation}

$S \widehat{N} R=10 \log \frac{\hat{A}^{2}}{\hat{\sigma}_{\mu}^{2}(k)} \quad$ with reference to $[1,14]$

Where $\hat{A}^{2}$-Estimated signal amplitude

$$
\begin{aligned}
& \hat{\sigma}_{\mu}^{2}(k)=\hat{d}^{2}(k)-\boldsymbol{H} \boldsymbol{P}(\boldsymbol{k} / \boldsymbol{k}) \boldsymbol{H}^{\boldsymbol{T}} \\
& \hat{d}(k)=\text { median } \frac{\mid \widehat{\mu}(k)-\text { median }(\hat{\mu}(k)) \mid}{0.6745} \\
& \hat{\mu}(k)=Y(k)-\boldsymbol{H} \widehat{\boldsymbol{Z}}(\boldsymbol{k} / \boldsymbol{k})
\end{aligned}
$$

Fig.3 shows the graph on window length verses cee for different acceleration at constant 20dB SNR. Window length which has less estimation error is taken as the optimal window length. From the graph it is observed that the optimum window lengths depend on the acceleration of the object. When same responses were observed at different SNRs, optimum window lengths are different for the same acceleration (a) $[1,14]$.Thus, the optimum window length selection depends on acceleration and SNR. For a $>10 \mathrm{~m} / \mathrm{s}^{2}$ and $\mathrm{SNR}>10 \mathrm{~dB}$, the window length variation is rather linear and linear interpolation technique is used to get optimum window length.

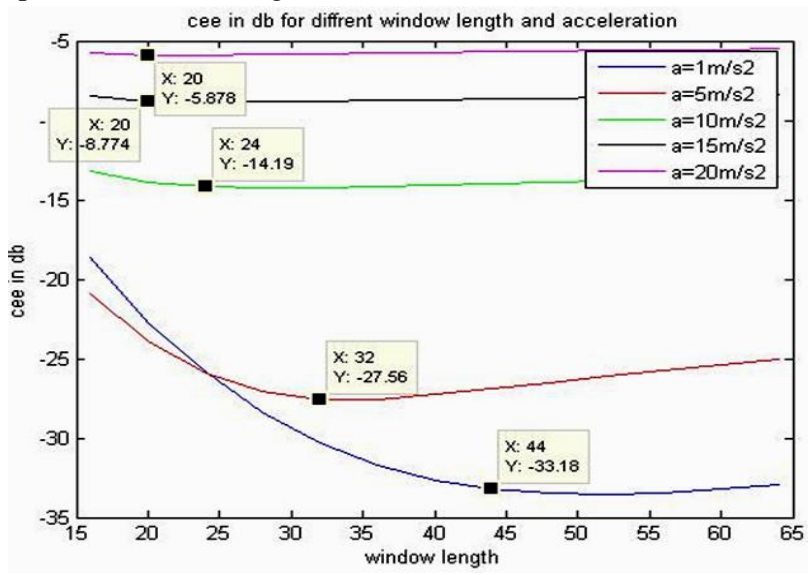

Fig.3 Window length verses cumulative estimation error for different acceleration at 20dB SNR

\section{KALMAN FILTER WITH STATE VECTOR FUSED VELOCITY ESTIMATION}

Two sensor information pertaining to the same target can be fused together to get the optimized information as shown in Fig.4

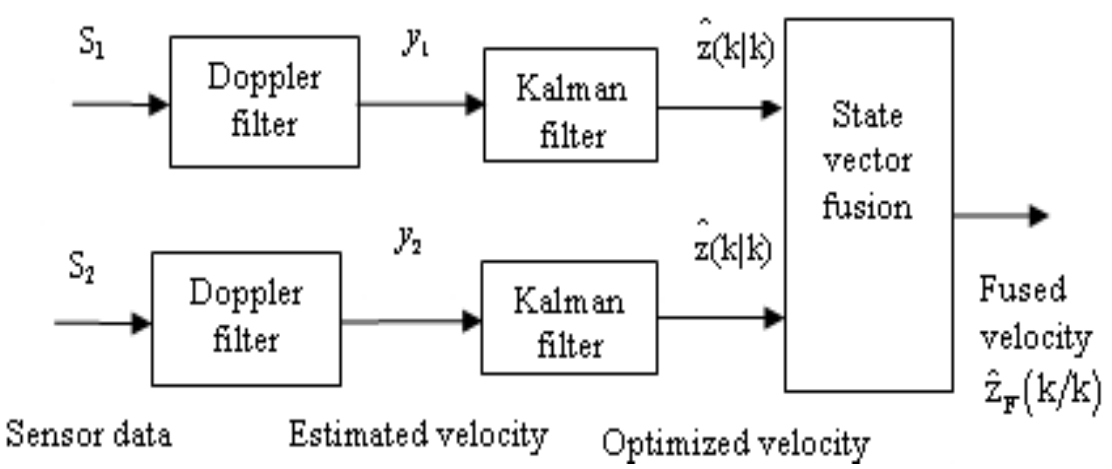

Fig. 4 Block diagram of non adaptive Kalman filter with state vector fusion

\subsection{Fusion technique}

Two commonly employed multi sensor information fusion [10] techniques are (i) state-vector fusion and (ii) measurement fusion $[3,5]$. The state-vector fusion method uses covariance of the filtered output of individual noisy sensor data to obtain an improved joint state estimate. On the other hand, the measurement fusion method directly fuses the sensor measurements to obtain a weighted or combined measurement and then uses a single Kalman Filter to obtain the final state estimate based on the fused measurement [3]. In the present case, state-vector fusion methodology has been employed.

\subsubsection{State vector fusion algorithm}

The fused state vector is given by [2], [3], [5].

$Z_{F}(k / k)=Z_{1}(k / k)+P_{1}(k / k) P_{E}^{-1}(k / k)\left[Z_{2}(k / k)-Z_{1}(k / k)\right](15)$ Where $\mathbf{Z}_{\mathbf{1}}(\boldsymbol{k} \mid \boldsymbol{k})$ and $\mathbf{Z}_{\mathbf{2}}(\boldsymbol{k} \mid \boldsymbol{k})$ are the estimated states of individual sensors and $\boldsymbol{P}_{E}(\boldsymbol{k} / \boldsymbol{k})$ is the error covariance which is given as,

$P_{E}(k / k)=P_{1}(k / k)+P_{2}(k / k)$

The covariance of the fused state vector is given by

$P_{F}(k / k)=P_{1}(k / k)-P_{1}(k / k) P_{E}^{-1}(k / k) P_{1}(k / k)^{T}$ 


\section{ADAPTIVE KALMAN FILTER WITH STATE VECTOR FUSED VELOCITY} ESTIMATION

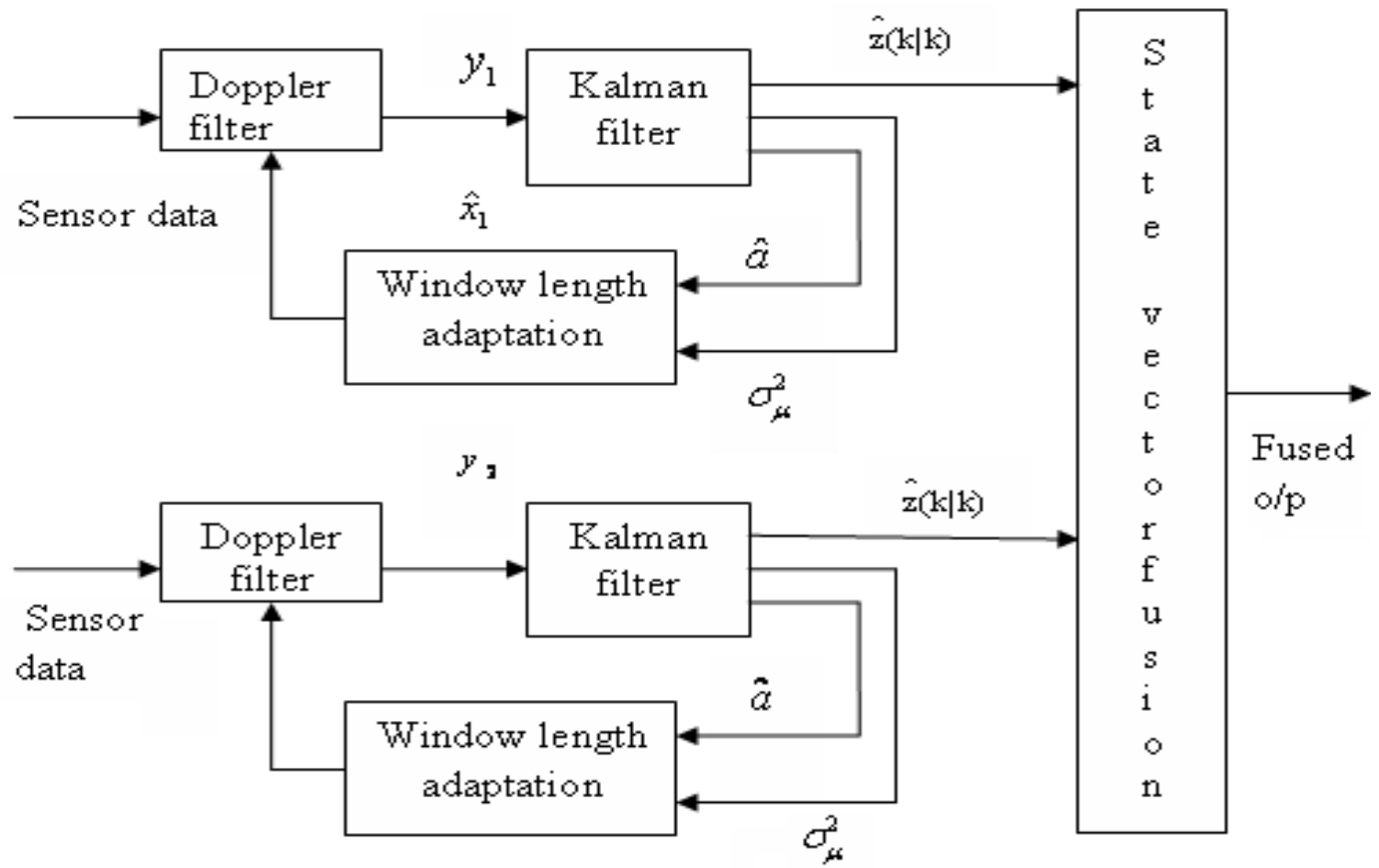

Fig. 5 Block diagram of adaptive Kalman filter with state vector

6. STATE VECTOR FUSED ADAPTIVE KALMAN FILTER VELOCITY ESTIMATION

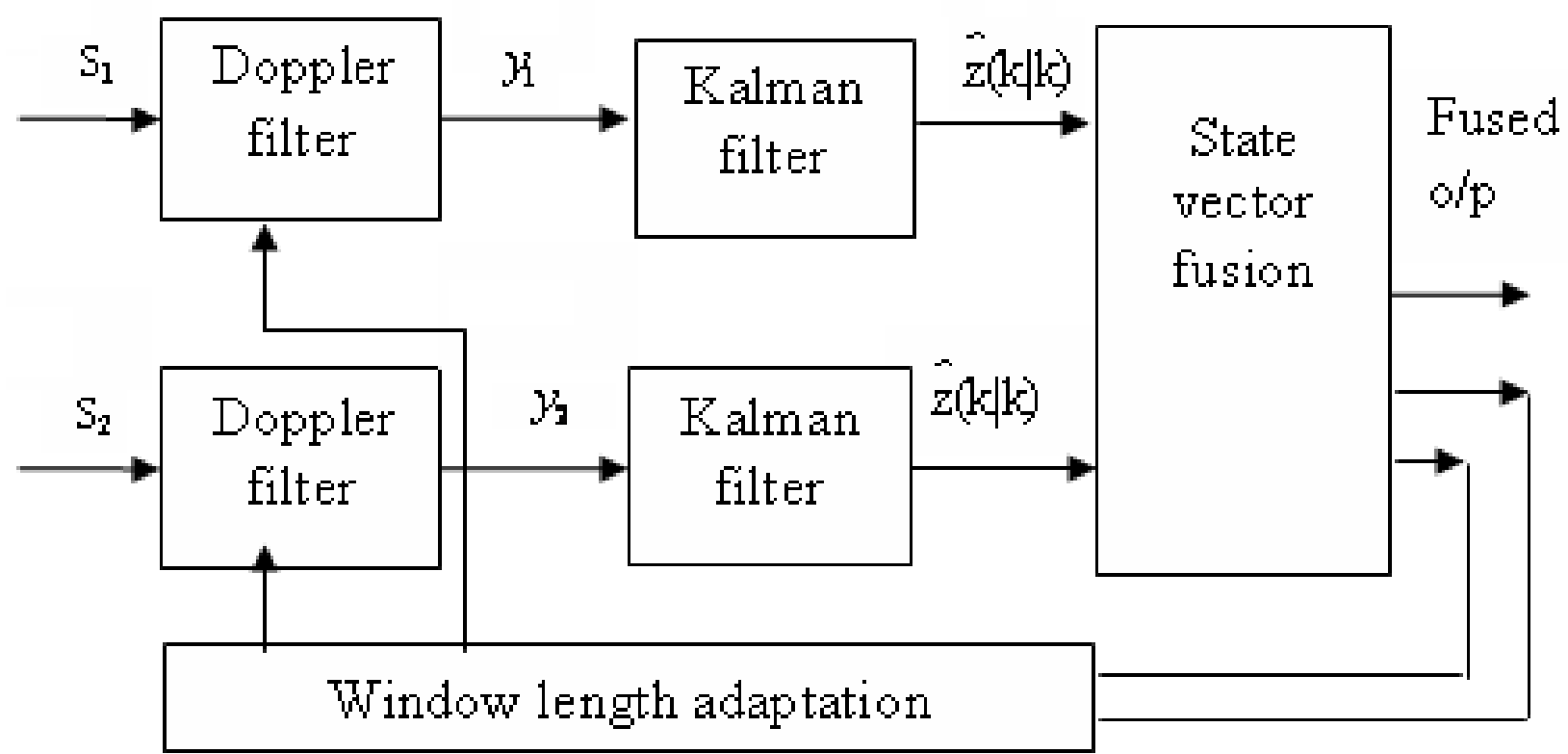

Fig. 6 Block diagram of state vector fused adaptive Kalman filter 


\section{Result and Discussion}

Here it is assumed that both the sensors received the data every 3 milliseconds. Maximum number of samples for processing is 5000. (i.e.) the period of observation is $5000 \times 3 \times 10^{-3}=15 \mathrm{~s}$.In the velocity profile, fast maneuvering, it is assumed to change from $250 \mathrm{~m} / \mathrm{s}$ to $116.6 \mathrm{~m} / \mathrm{s}$ within $3.6 \mathrm{~s}$. This gives the acceleration rate as $37.13 \mathrm{~m} / \mathrm{s}^{2}$.

First simulation is to compare the effect of Doppler Kalman filter with state vector fused Doppler Kalman filter.Fig.7,8,9 and 10 show the performance graphs of path 1. Fig. 11, 12 and 13 show the same analysis made for the path 2 .

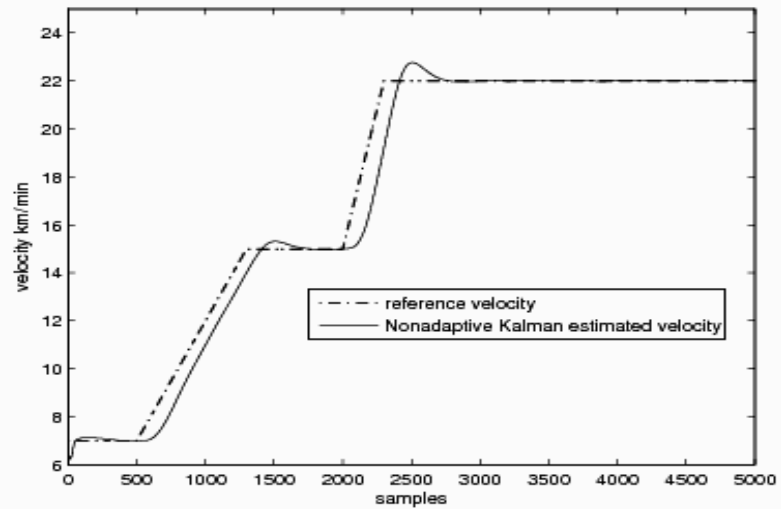

Fig.7 Kalman estimated velocity by sensor1 for path 1

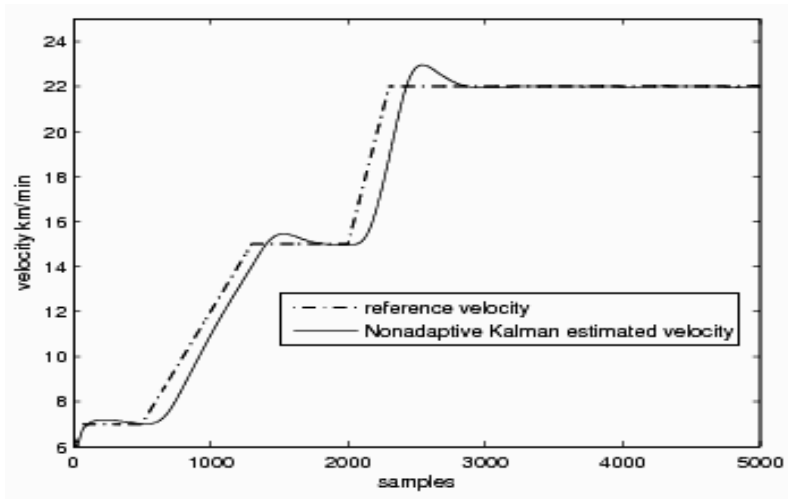

Fig.8 Kalman estimated velocity by sensor2 for path 1

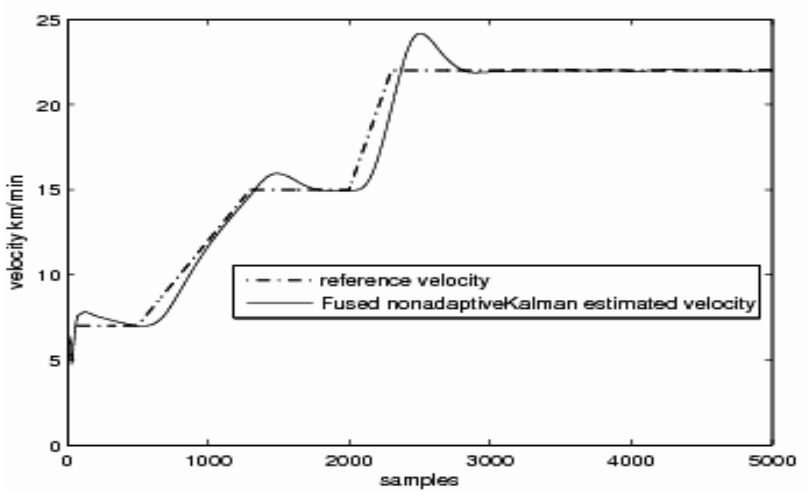

Fig.9 Fused Kalman estimated velocity by sensor $1 \& 2$ for path 1

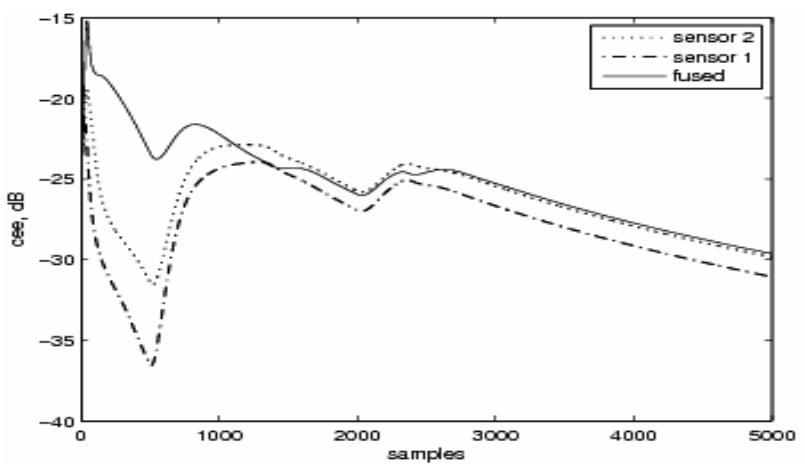

Fig.10 Cumulative estimation error (cee) in individual sensors estimation and fused output for path 1

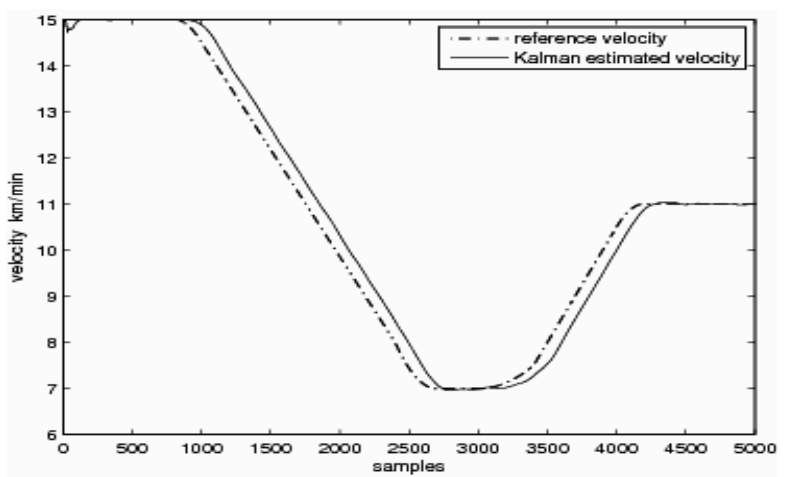

Fig.11 Kalman estimated velocity by sensor1 for path 2

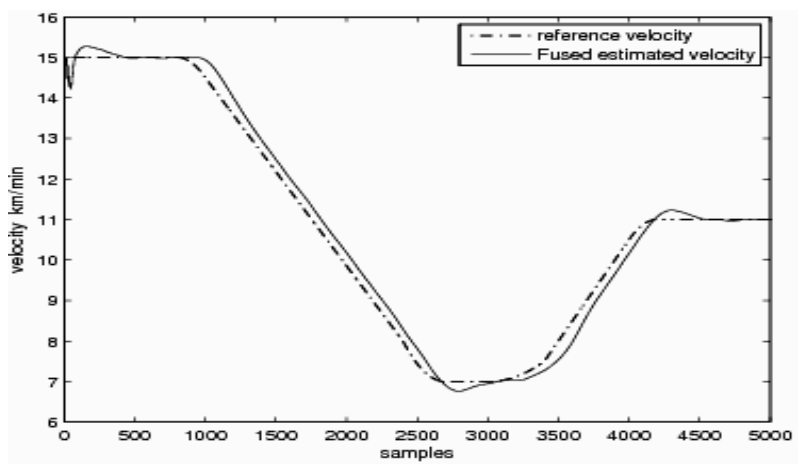

Fig.12 Kalman estimated velocity by sensor2 for path 2

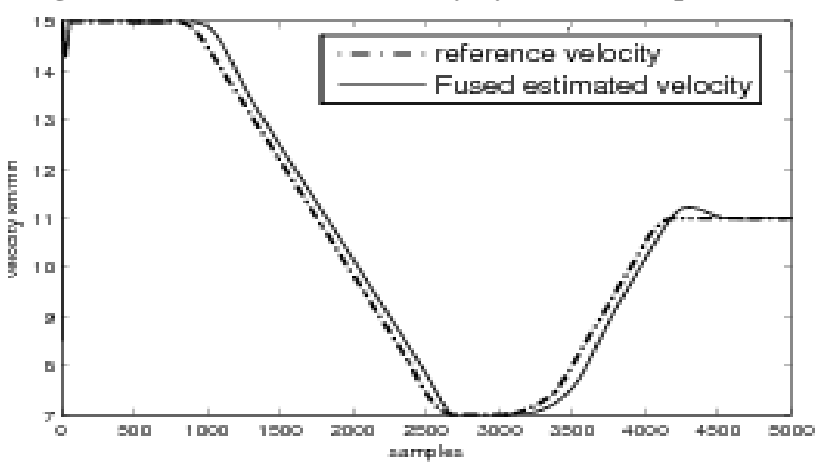

Fig.13 Fused Kalman estimated velocity by sensor 1\&2 for path 2 


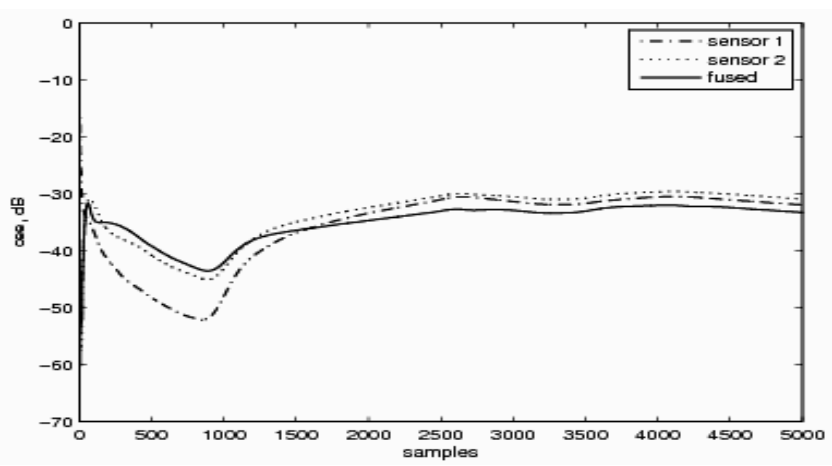

Fig.14 Cumulative estimation error (cee) in individual sensors estimation and fused output for path 2

From the above graph, it is obvious that fusion gives better velocity optimization in path 2 compared to path 1 . Figure 15 shows the comparative analysis of cumulative error in estimation using adaptive Kalman filter by the individual sensors with the fused adaptive Kalman technique (Fig.5) for path 2. Figure 16 shows the comparative analysis of error in estimation by the adaptive Kalman technique with that of adaptive fused Kalman (Fig.6).

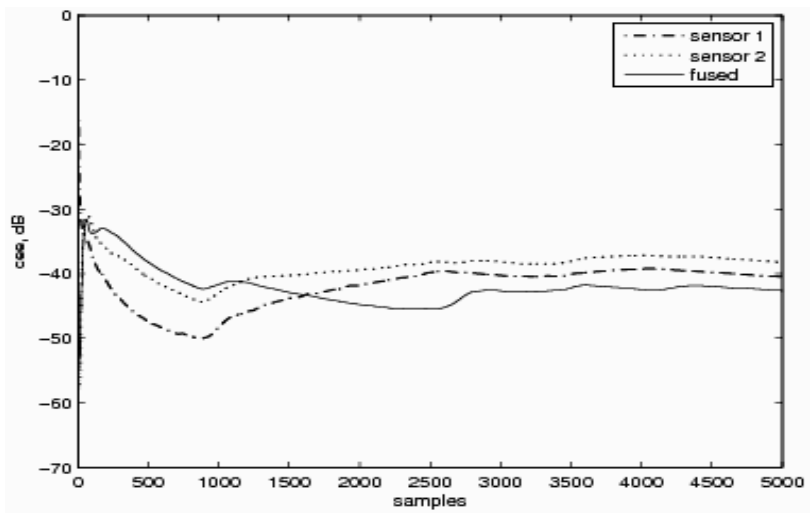

Fig.15. Estimation of error in adaptive Kalman filter by the individual sensors with the fused adaptive Kalman velocity

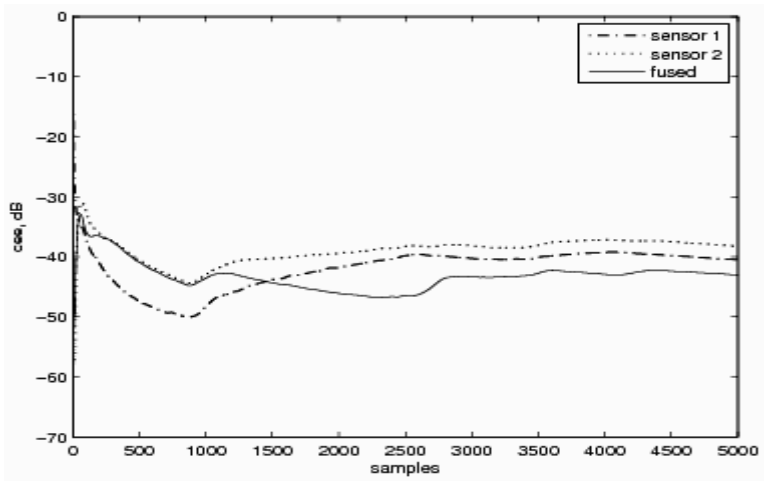

Fig.16. Estimation of error in adaptive Kalman filter by the individual sensors with the adaptive fused Kalman velocity estimation

\subsection{Result analysis}

From the results, for different SNR, delay in the Kalman smoothing due to the non adaptation, is minimized in the fused result. Over the entire SNR range, fusion gives the better result than the individual adaptive response at the acceleration and deceleration. At constant velocity individual adaptive estimation is better than the fused one. It is possible to estimate the velocity up to $15 \mathrm{~dB}$ SNR in both the paths by using both adaptive fusion and fused adaptation techniques. With single sensor information velocity estimation is possible only up to $35 \mathrm{~dB}$ SNR.

\section{CONCLUSION}

In the case of fast maneuvering (path 2), fusion gives better result than the individual sensor estimation. Individual adaptive estimation gives better results than the fused estimation only at constant velocity. For better smoothing, non adaptive fusion technique is best and for less delay in estimation, adaptive fusion or fused adaptation techniques are better. There has to be a tradeoff between the smoothing and the delay in estimation. Performance of the fused adaptation is same as that of the adaptive fusion. Fighter aircraft with the velocity of 1.7 mach and the maneuverability of $9 \mathrm{~g}$ to $-3.5 \mathrm{~g}\left(88.2 \mathrm{~m} / \mathrm{s}^{2}\right.$ to $\left.34.3 \mathrm{~m} / \mathrm{s}^{2}\right)$ may be tracked using these algorithms with reliable results.

\section{REFERENCES}

[1] V.D. Papic, Z.M. Djurovic and B.D. Kovacevic,“" Adaptive Doppler-Kalman filter for radar systems" IEE Proc.-Vis. Image Signal Process., Vol. 153, No. 3, June 2006

[2] G Girija, J R Raol, R Appavu Raj And Sudesh Kashyap, "Tracking filter and multi-sensor data fusion", Sadhana, Vol. 25, Part 2, April 2000, pp. 159-167.

[3] Shrabani Bhattacharya and R Appavu Raj,"Performance evaluation of multi-sensor data fusion technique for test range application", Sadhana Vol. 29, Part 2, April 2004, pp. 237-247

[4] Oppenheim, A V and Schafer R W, 1999. Discrete-time signal processing, 2nd Edition, Prentice-Hall.

[5] J. K. Hedrick J. Janga. Potier, 2004. Cooperative MultipleSensor Fusion for Automated Vehicle Control. Research Reports, University of California, Berkeley

[6] Ren C. Luo, Chih-Chen Yih, and Kuo Lan Su, "Multisensor Fusion and Integration: Approaches, Applications, and Future Research Directions", IEEE Sensors Journal, vol. 2, no. 2, April 2002

[7] Vladimir Katkovnik and L Jubisa Stankovic, "Instantaneous frequency estimation using Weigner distribution with varying and data driven window length", IEEE Trans. on Signal Processing, vol.46.no.9 Sept. 1998

[8] L. Jubisa Stankovic, Vladimir Katkovnik, "Algorithm for the Instantaneous Frequency Estimation Using Time Frequency Distributions with Adaptive Window Width", IEEE Signal Processing letters, vol. 5, no. 9, September 1998

[9] P.Banerjee and R.Appayu Raj, 2002. Multi-sensor Data Fusion Strategies for Real-Time Application in Test and Evaluation of Rockets / Missiles System, Industrial 
Technology 2002. Proceedings of IEEE International Conference.

[10] Yaakov Bar-Shalom, X.-Rong Li, Thiagalingam Kirubarajan, 2001, Estimation with Applications to Tracking and Navigation, A Wiley-Interscience Publication, John Wiley \& Sons, Inc.

[11] Merrill Skolnik, 2001. Introduction to Radar Systems, McGraw-Hall, New York, ISBN 0-07-290980-3

[12] Gan, Q., Harris, C.J., "Comparison of two measurement fusion methods for Kalman-filter-based multisensor data fusion", IEEE Transactions on Aerospace and Electronic Systems, Jan 2001

[13] Toshio Furukawa, Fumiko Muraoka and Yoshio Kosuge, 1998. Multi-Target and Multi-Sensor Data Fusion by Rule Based Tracking Methodology, SICE '98.

[14] V.D. Papic, Z.M. Djurovic and B.D. Kovacevic, 2002. Adaptive Doppler Filters Using Simultaneous Estimation of Target Acceleration and Signal to Noise Ratio, 10th Telecommunications forum TELFOR'2002, Belgrade, Yugoslavia. 